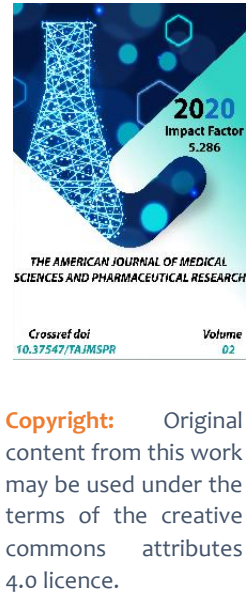

\title{
Optimization Of Pelvic Prolaps Surgical Correction Using Its Own Tissues
}

\author{
Shavkatov Hasan Shavkatovich \\ Assistant The Department Of Obstetrics And Gynecology, Samarkand State Medical Institute, \\ Uzbekistan \\ B.B. Negmadjanov \\ Head Of The Department Of Obstetrics And Gynecology, Samarkand State Medical Institute, \\ Uzbekistan
}

\section{ABSTRACT}

Pelvic organ prolapse (PTP) - pelvic floor and organ omission syndrome pelvic floor in isolation or in combination, which is extremely negatively affected by the quality of life of patients. According to world data, between 2.9 and 53\% of women report some form of PTP. Up to $47 \%$ of pelvic organ prolapsed patients are women of working age. According to the Women Health Initiative Study, among 16,616 women of perimenopaus age, the incidence of uterine prolapsed was $14.2 \%$, cystocele was $34.3 \%$, and reconcile was $18.6 \%$ In most cases, PTP is almost asymptomatic, which indicates its greater prevalence in the population.

\section{KEYWORDS}

Pelvic organ prolapsed, colpoperine-elevoropopplasty, the cervical stump.

\section{INTRODUCTION}

A special problem is GHG after a hysterectomy. According to the literature, the rate of vaginal dome drop after hysterectomy reaches $43 \%$. The disease has a wide age range, in addition, in recent years there has been a noticeable "rejuvenation" of this pathology, an increase in the number of complicated and recurrent forms. To reduce the recurrence rate after traditional vaginal correction, operating gynecologists began to use mesh implants to improve support of pelvic floor structures. It has long been known the high efficiency of the 
use of synthetic implants in the correction of groin hernia, abdominal sacropexia, etc. Current data on pelvic floor surgery show that the use of mesh implant in pelvic floor surgery has not only positive results, but also specific complications. Operations are often associated with the risk that has been actively discussed recently. Specific complications arising after correction using so-called mesh (eng, mesh) include mesh protrusion in the vagina (erosion) (erosion) (erosion) (erosion) (erosion) in the bladder, intestines, the development of infection, the appearance of pain syndrome, dyspareunia, urinary disorders and other pelvic dysfunctions. Extremely dangerous infectious complications include isolated cases of pelvic abscesses and fasciitis, recto-vaginal and vaginal fistulas, as well as the formation of coarse scars in the implantation area. These rare complications occur in less than 3\% of patients, but can lead to a significant decline in quality of life. According to the literature, the recurrence of PTO most often occurs in women after the correction of cystocele (8.9) as well as in patients with the 3 rd stage of PTO (cysto-or rectocele).

The problem does not lose its relevance. Due to changes in the demographic situation, we can expect a progressive increase in the number of patients with pelvic floor dysfunction, because older and older women are the fastest growing segment of the population. In addition, there has been an increase in the number of complicated and recurrent forms of this pathology.

This points to the need to study women's reproductive behavior after surgical treatment of genital prolapse applications of highly effective contraceptive methods.
Improving the effectiveness of the new modification of surgical treatment in patients with prolapse genitalia of varying severity and prevention of recurrence of the disease.

\section{MATERIALS AND METHODS OF THE STUDY}

A survey of 53 patients, which 21 patients operated on for genital prolapse, and 32 in the first were observed $\mathrm{GHG}$ s with different clinics, which were enrolled in the planned gynaecological department of the maternity complex No.3 in Samarkand between 2017 and 2020.

All surveyed were divided into two groups: (39.6\%) and the group were divided into two groups. women who were operated on for genital prolapse and had a complication, which was a comparison group, and (60.4\%) women who during the surgical treatment of genital prolapse, the provia of the moderation of surgical cortexing - the main group.

The average age in the 1st group was 58.9 years, in the 2nd group - 46.8 years.

All women under observation have been examined for women's clinical and a-levels. General examination, external and internal obstetric examination, clinical tests of blood, urine, determined the group and rhesus belonging of blood, examined the blood on Wasserman's reaction, conducted bacterioscopic and bacteriological examination of the contents of the cervix and vagina, urine analysis on Nechiporenko. In the study, the main group (61.9\%) patients are diagnosed with different forms of inflammatory processes (cervicitis, endocervicitis...) although the clinical picture dominated the symptoms of GHG. It is worth noting that in our patients 1st group with

THE AIM OF THE STUDY 
recurrent prolapse the period of relapse is 1-4 years.

The quality of life of patients with genital prolapse and surgical cortex with new modifications was assessed 3 years after the surgical treatment.

\section{THE RESULTS OF THE STUDY}

The choice of tactics of management, volume and access of surgical treatment of patients with female genital mutilation, as well as an objective assessment of the effectiveness of its results and postoperative rehabilitation was carried out on the basis of data history, clinical, laboratory, ultrasound and complex ugly methods of study. Regardless of volume and access, the operation aimed at eliminating the prolapse of the genital organs, including the complicated incontinence of urine as the main or one of the components included the correction of the insolvency of the pelvic floor muscles with the obligatory narrowing of the genital slit.

In the group of the main 32 patients underwent a surgical correction, with a new modification. After a transvaginal hysterectomy, the vaginal stump to the abdominal cavity is left open, by stitching the pelvic peritoneum along with the stumps of ligaments to the proximal wall of the vagina " $x$ " shaped like vicril seams, without peritonization, which provides reliable hemostasis and adequate pelvic drainage, guaranteeing the prevention of postoperative cultitis and infiltration, pelvic abdominal inflammation and contributing to the improvement of tissue repair and scaring conditions as well as recurrence of the prolapse of the vaginal walls. In this case, enterocele does not develop. Then we produce colpoperine-elevoropopplasty on the technique. During the inspection after 12 months, the height and depth of the vagina, the condition of scars, the tone of vaginal muscles, urinary function, the condition of the cervix were assessed.

For a comparative assessment of the long-term results of surgical treatment of AFP in patients of the main group, urination was monitored, the volume of the cervical stump was determined, the total length of the vagina, genital slit and the height of the vagina were measured.

According to the examination, after 12 months, there was a normal architectonics of the perineum and vagina, the cervical stump, which looked healthy in all patients.

This behavior of the woman was also explained by the fear of "disrupting the effect of surgical treatment." There were significantly fewer negative emotions during intimacy. Women noted an increase in the frequency of experienced orgasms, arousal by a sexual partner, and sexual desire. All of these changes led to an increase in satisfaction with sex life, which indicates a positive impact on the quality of life. 
Indicators of women with genital prolapse in the main group after surgery with a new modification.

\begin{tabular}{|c|c|c|}
\hline \multicolumn{1}{|c|}{ Indicators } & $\begin{array}{c}\text { Before } \\
\text { surgery }\end{array}$ & After 1 year \\
\cline { 2 - 3 } & \multicolumn{2}{|l|}{} \\
\hline General health & 15 & 32 \\
\hline Physical functioning & 11 & 29 \\
\hline Social functioning & 15 & 31 \\
\hline Vitality (according to subjective feelings) & 9 & 28 \\
\hline Self-reported mental health & 7 & 32 \\
\hline
\end{tabular}

One year later, we observed unfavorable outcomes of AVPO correction in 1 patients with a recurrence of I degree of prolapse of the anterior vaginal wall, which did not require surgical correction.

\section{CONCLUSIONS}

The features of the postoperative course and long-term results of surgical correction of genital prolapse indicate the high efficiency of the proposed method of surgical correction of genital prolapse and ensured a 1.5-fold reduction in the risk of infiltration and incision of postoperative sutures, a reduction in the length of hospital stay by 1.45 days and the risk of development. recurrences of the disease in the long-term follow-up by 1.6-2.8 times.

\section{REFERENCES}

1. Krasnopolsky V. I., Buyanova S. N., Petrova V. D. Combined treatment patients with prolapse and prolapse of internal genital organs and burning urine with the use of anti-stress technologies.whose. - M., 2003 .- $41 \mathrm{p}$.

2. Hendrix S. L., Clark A., Nygaard I. et al. Pelvic organ prolapse in the Women "s Health Initiative: gravity and gravidity. Am J Obstet Gynecol 2002; 186 (6): 1160-

3. Thakar R., Stanton S. Management of genital prolapse. BMJ 2002; 324 (7348): 1258-62.

4. Clemons, J.L. Impact of the 2011 FDA Transvaginal Mesh Safety Update on AUGS Members' Use of Synthetic Mesh and Biologic Grafts in Pelvic Reconstructive Surgery / J.L. Clemons, M. Weinstein, M.K. Guess, M. Alperin, P. Moalli, W.T. Gregory, E.S. Lukacz, V.W. Sung, B.H. Chen and C.S. Bradley. // AUGS Research Committee 
Female Pelvic Med Reconstr Surg. - 2013. N.19. - R.191-198.

5. Clemons, J.L. Impact of the 2011 FDA Transvaginal Mesh Safety Update on AUGS Members' Use of Synthetic Mesh and Biologic Grafts in Pelvic Reconstructive Surgery / J.L. Clemons, M. Weinstein, M.K. Guess, M. Alperin, P. Moalli, W.T. Gregory, E.S. Lukacz, V.W. Sung, B.H. Chen and C.S. Bradley. // The Journal Of Urology. - 2014. Vol. 191. - P.1051-1059.

6. Haylen, B.T. International Continence Society (ICS) joint terminology and classification of the complications related directly to the insertion of prostheses (meshes, implants, tapes) \& grafts in female pelvic floor surgery / B.T. Haylen, R.M. Freeman, S.E. Swift. // An International Urogynecological Association (IUGA) // Neurourol. Urodyn. 2010. - Vol.29, N 1. - R.4-20.

7. Gvozdev, M. Yu. The first experience of using in Russia non-malignant mesh technologies in the treatment of patients with pelvic prolapse / M.Yu. Gvozdev et al. // Russian Bulletin of ObstetricianGynecologist. - 2012. - No. 5. - S. 57-63.

8. Huser, M. Outcomes of anterior vaginal wall repair using polypropylene mesh / $M$. Huser et al. // Česka Gynecol. - 2012. - Vol.77, N.5. - R.407-413.

9. Apolikhina I.A., Ibinaeva I.S., Zheleznyakova A., Saidova A.S.Standardization of diagnostic approaches to the management of women with different types of urinary incontinence // Obstetrics and gynecology. 2009. No. 1. P. 67-71.

10. Buyanova S.N., Savelyev S.V., Grishin V.L., Senchakova T.N. Principles selection of surgical correction method for genital prolapse and incontinence urine // Russian
Bulletin of Obstetrician-Gynecologist. 2003. No. 3. P. 34-39.

11. Krasnopolsky V.I., Buyanova S.N., Shchukina N.A., Popov A.A. Operative gynecology. 3rd ed. I IN AND. Krasnopolsky, S.N. Buyanova, N.A. Shchukina, A.A. Popov, M .: MEDpressinform :, 2017.320 p.

12. Ishchenko A.A., Ishchenko A.I., Alexandrov L.S., Gorbenko O.Yu., Khokhlova I.D., Gavrilova T.V., Gadaeva I.V. Transvaginal surgical prevention of posthysterectomy vaginal dome prolapse in patients with complete prolapse of the uterus // Questions of gynecology, obstetrics and perinatology. 2017. No. 3 (16). S. 43-46. 